\title{
5'-N-Ethylcarboxamido Adenosine Attenuates Myocardial Ischemia/Reperfusion Injury In Type 2 Diabetic Rats Through A2AR/PKCa/miR-15a Signaling
}

Chao Chen ( 2016283030160@whu.edu.cn )

The Affiliated Hospital of Guizhou Medical University

Jianjuan Ke

Wuhan University Zhongnan Hospital

Huang Ding

Wuhan University Zhongnan Hospital

Chengjun Hu

Wuhan University School of Basic Medical Sciences

\section{Zhenggang Wang}

Wuhan University Zhongnan Hospital

Mengyun Li

Wuhan University Zhongnan Hospital

\section{Yanlin Wang}

Wuhan University Zhongnan Hospital

\section{Research Article}

Keywords: Adenosine, Myocardial infarction, Apoptosis, PKCa, MicroRNA-15, Bcl2

Posted Date: September 15th, 2021

DOl: https://doi.org/10.21203/rs.3.rs-872350/v1

License: (c) (1) This work is licensed under a Creative Commons Attribution 4.0 International License. Read Full License 


\section{Abstract}

Background/aims: Type 2 diabetes mellitus aggravates myocardial ischemia/reperfusion injury (MI/RI). Activation of adenosine receptors (ARs) confer to attenuated MI/RI in nondiabetic animals and human. However, this effects and mechanism of ARs in the type 2 diabetic state are still unknown. In present study, we established a type 2 diabetic rat in vivo myocardial ischemia/reperfusion (MI/R) model to evaluate the effect of ARs on MI/RI with a focus on the A2A adenosine receptor (A2 AR) -mediated cardioprotective effects.

Methods: Type 2 diabetic rat were subjected to myocardial infarction by LAD ligation in situ and randomly received ARs agonist and/or antagonists or vehicle treatment. After $2 \mathrm{~h}$ marker of the extent of myocardial damage(ejection fraction of the LV, Infarct size, plasma cardiac troponin I) were measured and pro- and anti-apoptotic signals (protein kinase $\mathrm{Ca \bigotimes Bcl-2,Bax,} \mathrm{miR-15),} \mathrm{and} \mathrm{marker} \mathrm{of} \mathrm{apoptosis}$ execution (cleaved caspase-3, TUNEL) were quantified in the infarcted myocardium.

Results: non-selective adenosine receptor agonist 5'-(N-ethylcarboxamido) adenosine treatment attenuates $\mathrm{MI} / \mathrm{RI}$, improve post-MI/R left ventricular function, limit infarct size, reduce cardiac troponin I release, reduce myocardial apoptosis, up-regulates bcl2 and down-regulates miR-15a, bax and cleaved caspase-3 expression; This protective effects were attenuated by pretreatment with selective A2AR antagonist ZM241385 or PKCa-selective inhibitor Go6976; and duplicated by treatment with A2ARselective agonist CGS21680 or PKCa-potent activator PMA.

Conclusions: NECA reduces MI/RI in T2DM rats via the A2AR/PKCa/miR-15a signaling pathway; NECA is a useful target candidate for the treatment of $\mathrm{MI} / \mathrm{RI}$ in patient with type 2diabetes.

\section{Introduction}

Myocardial infarction(MI)is a major perioperative complication in patients with type 2 diabetes mellitus(T2DM) [1, 2].. The relative risk of ischemic heart disease in diabetic patients is more than two times that in the nondiabetic population[3]. Clinical and epidemiological studies indicate that patients with diabetes have an increased mortality rate after MI compared with nondiabetic patients [4]. T2DM was also confirmed to aggravated $\mathrm{Ml} / \mathrm{RI}$ in predominant animal experiments. Timely reperfusion remains the major treatment for acute $\mathrm{MI}$ to salvage the ischemic myocardium, leading to improved residual ventricular function and clinical outcomes, but reperfusion itself may cause reversible or irreversible tissue injury, termed "myocardial ischemia /reperfusion injury" (MI/RI). The pathogenesis of MI/RI is multifactorial, and myocardial apoptosis is one of the major pathogenic mechanisms. apoptosis reduces the number of cardiomyocytes to impair cardiac systolic function. Further, T2DM also exaggerates cardiomyocyte apoptosis during ischemia-reperfusion, alleviation of cardiomyocyte apoptosis during ischemia-reperfusion may be an alternative method to improve post ischemia cardiac function[1, 2].

Adenosine, an endogenous purine nucleoside, shows increased concentrations in the extracellular space during MI[5]. In nondiabetic subjects, sufficient evidence indicates that adenosine can exert multiple 
beneficial effects during $\mathrm{Ml} / \mathrm{R}$ by binding to four $\mathrm{G}$ protein-coupled receptors known as $\mathrm{A} 1$ adenosine receptors(A1Rs), A2A adenosine receptors (A2ARs), A2B adenosine receptors (A2BRs) and A3 adenosine receptors (A3Rs), all of which were expressed in mammalian ventricular myocardium[6]. A2AR plays a critical role in adenosine cardioprotective effects during early reperfusion, and pharmacological and genetic evidence has demonstrated that A2ARs are important to protection organs against apoptosis during reperfusion $[7,8]$. However, the T2DM heart is resistant to cardioprotective strategies such as ischemic preconditioning/postconditioning and remote ischemic conditioning[9]. whether the cardioprotective effects of A2AR activation are present in T2MD conditions is unknown, if so, what is the mechanism?

Protein kinase $\mathrm{C}$ isozymes (PKCs) are a family of serine/threonine kinases that are mediated diversely physiological processes by regulated signal transduction[10]. Ischemic preconditioning (IPC) and ischemic postconditioning (IPO) exert potent effects of against apoptosis on the I/R heart by activating PKCs[11, 12]. PKCa, a conventional isoforms, is the predominant subtype expressed in most mammalian hearts[10]and plays an principal role in attenuation of cardiomyocytes apoptosis[13, 14].Interestingly, activation of adenosine receptors is a requirement for both IPC and IPO to reduce infarct size in early reperfusion[11]. Subsequent studies demonstrated PKCa is a downstream signal in the adenosine's cardioprotective signaling pathway[15], but, the mechanism of $\mathrm{PKCa}$ against cardiomyocyte apoptosis is still unclear.

MicroRNAs (miRs) are a family of small non-coding RNAs with typically 22 nucleotides in length that negatively regulate mRNA translation by base pairing with the 3 ' untranslated regions (UTRs) of messenger RNAs (mRNA). The miR-15 family consists of miR-15a, miR-15b, miR-16, miR-195, and miR497 , and is upregulated during MI/RI[16, 17]. These miRNAs are confirmed to promote cardiomyocytes apoptosis by inhibiting the expression of pro-survival proteins Bcl-2[18, 19]. Studies have shown that inhibiting the expression of miR-15a ameliorated cardiomyocytes apoptosis by restored Bcl-2 expression[19]. Interestingly, Cohen et al.[20] and Brandenstein et al.[21, 22]found that the PKCa downregulation of miR-15a by binding directly to pri-miRNA-15a in the nucleus and suppresses the release of pri-miRNA-15a, leading to decreased mature cytoplasmic miRNA-15a levels. Therefore, we presumed that PKCa regulates cardiomyocytes apoptosis by down-regulation the expression of miR-15a.

Taken together, in present study we hypothesized that 5'-N-ethylcarboxamido adenosine attenuate MI/RI and cardiomyocytes apoptosis through A2AR/PKCa / miR-15a signaling pathway

\section{Materials And Methods}

\subsection{Animals and T2MD model}

Male Sprague Dawley (SD) rat were purchased from the Hubei Provincial Center for Disease Control and Prevention and utilized at 200 220 grams of weight (age $8 \sim 9$ weeks). The animal experimental protocols were approved by the Experimental Animal Center of Zhongnan Hospital. All animal 
experiments were performed under the Guidelines on Humane Use and Care of Laboratory Animals for Biomedical Research published by the National Institutes of Health (No. $85-23$, revised 1996).

T2MD animal model induced by the strategy of high fat diet and intraperitoneal injection of streptozotocin. After the 1-week acclimation period, rats were fed with containing $60 \%$ fat (as a percentage of total $\mathrm{kcal}$ ), $20 \%$ carbohydrates, and $20 \%$ proteins animal-feeding for 2 weeks. Then, intraperitoneal injected streptozotocin $35 \mathrm{mg} / \mathrm{kg}$ [23]. After $72 \mathrm{~h}$, Tail vein random blood glucose were measured. Diabetes was confirmed by the presence of hyperglycemia (>16.7 mmol/l). To simulate the physiology of chronic diabetes, we continuously fed rats with high-fat food for 12 weeks and tested the random blood glucose once a week via the tail vein to confirm the success of the chronic diabetes model. The blood glucose and body weight data of all rats are shown in Figure S1 of the supplements.

\subsection{Myocardial infarction protocol}

The MI/R procedure was carried out as described previously[24]. Intraperitoneal anesthesia with $3 \%$ sodium pentobarbital $(60 \mathrm{mg} / \mathrm{kg}$ ) was followed by tracheotomy. Respiratory support was accomplished by tracheal intubation and a rodent ventilator (HX-100E; Taimeng Tech Co, Ltd, China). The rats were ventilated with air under a tidal volume set as $2 \sim 3 \mathrm{ml} / 100 \mathrm{~g}$, a frequence of $60 \sim 80$ breaths perminute, and a inspiratory/expiratory ratio as 1:1. An adjustable heating pad (JR-10-30; Taimeng Tech Co., Ltd., China) was used to keep the body temperature between 36 to $37^{\circ} \mathrm{C}$. The cannulation of left femoral vein and right femoral artery were performed respectively to inject either drug treatments or lactated Ringer's solution with a microinfusion pump (WZ-50S, Medical Instruments of Zhejiang University, China) and to monitor blood pressure and heart rate(HR) continuously (BL-420N; Taimeng Co., Ltd., China). After a left lateral thoracotomy and a pericardiotomy, rats were submitted to $30 \mathrm{~min}$ of left anterior descending artery (LAD) occlusion followed by $2 \mathrm{~h}$ of reperfusion. Ischemia was verified through a blood pressure decreased transiently in and the occurrence of cyanosis on the myocardial surface. Reperfusion was confirmed by epicardial hyperemic response and electrocardiogram present ischemic repolarization changes (ST-segment elevation and fall) during coronary occlusion and reperfusion.

\subsection{Experimental groups}

After 12 weeks of the diabetes model induction, rats were submitted to 30 min of LAD occlusion followed by $2 \mathrm{~h}$ of reperfusion as described above and randomly received agonist and/or antagonists or vehicle treatment. As shown in (Fig. 1): (1): In the I/R group, vehicle was infused over 20 min before 5 min of reperfusion; (2):in the NECA group, NECA, a non-selective but potent adenosine receptor agonist, $2 \mu \mathrm{g} / \mathrm{kg}$ was infused as a bolus dose before 5 min of reperfusion; after application of the bolus, $0.2 \mu \mathrm{g} / \mathrm{kg} / \mathrm{min}$ was maintained $60 \mathrm{~min}[25,26]$; (3):in the NECA + ZM group, NECA was applied as described above, and ZM241385(0.2 mg/kg intravenously), a selective A2AR antagonist, was administered before $5 \mathrm{~min}$ of NECA as a bolus to ensure proper receptor blockade as previously described[27]; (4):in the CGS group, CGS21680, a highly selective A2AR agonist, was given as a bolus of $30 \mu \mathrm{g} / \mathrm{kg}$ intravenously 5 min before the onset of reperfusion, followed by a $60-\mathrm{min}$ infusion of $3 \mu \mathrm{g} / \mathrm{kg} / \mathrm{min}[26]$; (5):in the NECA + Go group, NECA applied as described above, and Go6976, a selective PKCa inhibitor, was infused at $1 \mu \mathrm{M}$ in a total 
of $1 \mathrm{ml}$ over 10 min before 5 min of the NECA treatment ; (6): in the PMA group, PMA, a nonselective but potent PKCa activator, was infused at $0.5 \mu \mathrm{g} / \mathrm{kg} / \mathrm{min}$ beginning $5 \mathrm{~min}$ before reperfusion and extending for $20 \mathrm{~min}[25]$; (7): in the PMA + Go group, PMA was applied as described above, and Go6976 at $1 \mu \mathrm{M}$ was infused in a total $1 \mathrm{ml}$ over 10 min before 5 min of PMA treatment; (8): in the Sham group, same surgical procedures and vehicle treatment were performed apart from that the suture passed under the left coronary artery was kept untied.

NECA(1691), ZM241385(1036) and CGS21680(1063) were purchased from Tocris Bioscience (Ellisville, MO). Go6976 (S7119) was purchased from Selleck Chemicals (Shanghai, China). PMA were purchased from Beijing Solarbio Science \& Technology Co., (Beijing, China). DMSO was purchased from Sigma Chemical Co. (St. Louis, MO). All drugs were deliquescence in DMSO and further diluted in $1 \mathrm{ml} 0.9 \%$ saline before administrated to the rat, and the final concentration of DMSO was kept below $1 \%$; the vehicle was $1 \%$ DMSO in $1 \mathrm{ml} 0.9 \%$ saline. DMSO $<300 \mu \mathrm{l} / \mathrm{kg}$ had no effect in rat hearts, as shown by previous studies[27].

\subsection{Echocardiography}

After $2 \mathrm{~h}$ of reperfusion, cardiac function was evaluated by two-dimensional mode and motion (M)-mode echocardiography. As previously described[28] echocardiography was performed using an $11 \mathrm{MHz}$ linear transducer connected to an echocardiographic system (VIVID7, GE, USA). Briefly, M-mode images were obtained from the short axis view at the level of the papillary muscle to measure the left ventricular internal dimension systole (LVIDs) and left ventricular internal dimension diastole (LVIDd) and to calculate LV fractional shortening (LVFS, \%) using the following formula: ([LVIDd - LVIDs] × 100/LVIDd); the parasternal long and short axis at the level of papillary muscle 2D views was obtained to calculate the LV end-diastolic (LVEDV) and end-systolic (LVESV) volumes as well as the ejection fraction of the LV (LVEF) by computerized algorithms.

\subsection{Infarct size and apoptosis}

Following $2 \mathrm{~h}$ of reperfusion, the LAD was occlusion permanently, and 5\% Evans blue solution (E2129; Sigma) was injected into the venous line to distinguish the left ventricular (LV) area at risk (AAR) as described in our previous work[24]. A pentobarbital overdose was used to euthanize animals, the heart was immediately removed and rinsed in a $0.9 \%$ ice-salt mixture, and the atria and great vessels were excised. The sample was frozen in $-20^{\circ} \mathrm{C}$ for $45 \mathrm{~min}$ and cut into 5 slices ( $2 \mathrm{~mm}$ per slice) which were incubated in phosphate-buffered $1 \%$ triphenyltetrazolium chloride (TTC) (T8877; Sigma) (pH 7.4) in $37^{\circ} \mathrm{C}$ for 20 min. After incubation, each slice was then immersed in 10\% formalin to enhance the contrast between the stained (viable) and unstained (necrotic) tissues. A CCD digital camera took photos of each slice. Image-Pro Plus 6.0 software (Media Cybernetics, Inc, Washington Street, Rockville, MD) was used to quantify the total area, the ischemic area and the infarcted area of each slice. The AAR was devoid of Evans blue dye, whereas the infarcted tissue within the AAR was TTC-negative-stained. Infarct size was expressed as a percentage of infarct area (IF) over total AAR (IF/AAR×100\%). 
Analysis of myocardial apoptosis was realized by a terminal deoxynucleotidyl transferase-mediated dUTP nick end labeling (TUNEL) assay as previously study[29]. Briefly, cardiac tissue from the infarct zone was fixed in $4 \%$ paraformaldehyde $(\mathrm{pH} \mathrm{7.4)}$ ) for $24 \mathrm{hr}$. and then embedded in paraffin and sectioned (10 $\mu \mathrm{m}$ thickness). TUNEL staining was manipulated according to the manufacturer's recommendations (Roche Molecular Biochemicals, Mannheim, Germany). The TUNEL-positive cells with nuclei stained green and all cells with DAPI staining were counted within five randomly selected fields. The apoptosis index was expressed as the percentage of positively stained myocytes/ total myocytes counted $\times 100 \%$.

\subsection{Determination of plasma cardiac troponin I(cTnl)}

Measurement of plasma cTnl concentration was performed with enzyme-linked immunosorbent assays (ELISAs) as described in our previous study[24]. Samples of femoral vein blood (3 ml) were collected after $2 \mathrm{~h}$ reperfusion and centrifuged at $3000 \mathrm{rpm}$ for $15 \mathrm{~min}$ at $-4^{\circ} \mathrm{C}$ using a desktop centrifuge (TLG-6G, Shanghai Anting Scientific Instrument Factory, Shanghai, China). The plasma was subsequently extracted and stored at $-80^{\circ} \mathrm{C}$ until further use. A double-antibody sandwich ELISA kit (CSB-08594r; CUSABIO BIOTECH Co., Ltd., Wuhan, China) was used to detect the cTnl concentration.

\subsection{Western blotting}

After $2 \mathrm{~h}$ of reperfusion, rat infarct zone cardiac tissues samples were rapidly collected and snap-frozen in liquid nitrogen. Western blotting was conducted as in previous studies[24]. Momently, after the bicinchoninic acid (BCA) quantitative analysis, total protein was separated by $10 \%$ SDS-PAGE gels and then transferred to PVDF membranes which were blocked with $5 \%$ nonfat milk for $3 \mathrm{~h}$ at room temperature. Afterwards proteins were probed overnight at $4{ }^{\circ} \mathrm{C}$ with specific antibodies against PKCa (ab32376, Abcam;1:2000), p-PKCa (ab180848, Abcam;1:1000), Cleaved Caspase-3 (ab49822, Abcam;1:1000), Bcl-2 (ab196495, Abcam;1:2000), Bax (ab32503, Abcam;1:1000) and GAPDH (ab181602, Abcam;1:10000). The membrane was washed three times with TBST buffer and then incubated with a horseradish peroxidase-conjugated secondary antibody (As1107, Aspen; 1:10000) for $1 \mathrm{~h}$ at room temperature, and immunoreactive bands were detected by enhanced chemiluminescence (ECL). Quantitative assessment of band densities was performed densitometrically using ImageJ software.

\subsection{Isolation of RNA and qPCR assays}

Total RNA was isolated from the rat infarct zone cardiac tissues using TRIzol (Invitrogen, USA) according to the manufacturer's protocol. The cDNA was obtained using a Moloney Murine Leukemia Virus (M-MLV) Reverse Transcriptase Kit (Invitrogen, USA) according to the manufacturer's protocol. U6 was applied as an internal control. The real-time qRT-PCR analysis was performed on a StepOne Real-Time PCR systems (Invitrogen; USA) to examine the expression level of miR-15a, miR-15b, miR-16, miR-195 and miR-497 with SYBR ${ }^{\circledR}$ Premix Ex Taq ${ }^{\text {TM }}$ (TakaRa, China.) according to the manufacturer's protocol. Primer sequences were miR-15a: forward 5-TGGGCAGCACAT $\forall T G G \top T-3$, reverse 5

- CTC $\forall C T G G T G T C G T G G A G T C-3$; miR-15b: forward 5

- CGCAGCACATCATGG T TACA - 3, rever se5-CTCAACTGGTGTCGTGGAGTC-3 ; miR - $16: f$ or ward5-TCAGCACGTAAATATTGGCG-3, rever se5-CTCAACTGGTGTCGTGGAGTC-3 
; miR - 195: $f$ or ward5-CGCAGCACAGAAATATTGGC-3rever se 5-CTCAACTGGTGTCGTGGAGTC-3 ; miR $-497: f$ or ward5-TGCAGCACACTGTGGTTTGTA-3, rever se 5 -

CTCAACTGGTGTCGTGGAGTC-3; $U 6: f$ or ward5-CCTGCTTCGGCAGCACAT-3rever se 5AACGCTTCACGAATTTGCGT-3․ qPCR was initiated at $95^{\circ} \mathrm{C}$ for $1 \mathrm{~min}$, followed by 40 cycles of denaturation at $95^{\circ} \mathrm{C}$ for $15 \mathrm{sec}$, annealing at $58^{\circ} \mathrm{C}$ for $20 \mathrm{sec}$, and extension at $72^{\circ} \mathrm{C}$ for $45 \mathrm{sec}$. The $2^{-\triangle \Delta C T}$ method was used to calculate relative miRNA expression, and $\mathrm{U} 6$ served as an internal reference. $\Delta \mathrm{Ct}=\mathrm{Ct}_{\text {target gene }} \mathrm{Ct}_{\mathrm{U}}, \Delta \Delta \mathrm{Ct}=\mathrm{Ct}$ experiment group $-\mathrm{Ct}$ control group.

\subsection{Confocal imaging of PKCa localization in cardiomyocytes.}

After $2 \mathrm{~h}$ of reperfusion, the rat infarct zone cardiac tissues were fixed in $4 \%$ formaldehyde and paraffin embedded and $5 \mu \mathrm{m}$ sections were mounted on microscope slides. Immunodetection was performed as described previously[30]. Briefly, paraffin sections were deparaffinized in xylene and hydrated through a series of graded ethanol. After the cardiac sections were blocked with $5 \%$ bovine serum albumin (BSA), they were incubated in PBST overnight at $4^{\circ} \mathrm{C}$ with anti-PKCa primary antibodies (ab32376, Abcam). Then, sections were then labeled with Cy3-conjugated secondary antibody (as-1109, Aspen) for $1 \mathrm{~h}$ at $37^{\circ} \mathrm{C}$, and nuclei were counterstained with DAPI. Finally, immunofluorescence was visualized with confocal laser scanning microscopy (Olympus FV500).

\subsection{Electrophoretic mobility shift assay (EMSA).}

Nuclear proteins were extracted from the rat infarct zone cardiac tissues according to the manufacturer's protocol (Beyotime Biotech, Shanghai, China), and protein concentrations were determination by BCA assay. The sequences of the probes were determined in a previous study[21], wild-type pri-miR-15a (5'TGTGGATTTTGAAAAGG-3') and mutated pri-miR-15a (5'-TGTGGAAAGGGAAAAGG-3'), which were synthesized and labelled according to the project of Invitrogen Technology (Shanghai, China). EMSAs were executed as the specifications of chemiluminescent EMSA kit (Beyotime Biotech, Shanghai, China). Briefly, a standard reaction mixtures for EMSA contained $2 \mu \mathrm{g}$ nuclear protein, $200 \mathrm{fmol}$ of biotin-labeled annealed oligonucleotides, $2 \mu \mathrm{l}$ of $10 \times$ binding buffer $(100 \mathrm{mMTris}, 500 \mathrm{mM} \mathrm{KCl}$, and $10 \mathrm{mMDTT}$, pH 7.5), $1 \mu \mathrm{l}$ of $1 \mu \mathrm{g} / \mu \mathrm{l}$ poly (dl-dC), $1 \mu \mathrm{l}$ of $50 \%(\mathrm{v} / \mathrm{v})$ glycerol, $1 \mu \mathrm{l}$ of $1 \%(\mathrm{v} / \mathrm{v})$ Nonidet P-40, $1 \mu \mathrm{l}$ of $100 \mathrm{mM}$ $\mathrm{MgCl} 2,1 \mu \mathrm{l}$ of $200 \mathrm{mM}$ EDTA and double-distilled water to a final volume of $20 \mu \mathrm{l}$. To identify the specific RNA-binding complexes of PKCa, we added 40 pmol non-labeled("cold") wild-type or mutated probes to the reaction mixture 10 min before addition of the labeled probes.In addition, a PKCa antibody (Santa Cruz) was used for supershift EMSAs. After a 20 min incubation at room temperature, the RNA-protein complexes were loaded onto $6 \%$ nondenaturing polyacrylamide gels subjected to electrophoresis, and then transferred to $\mathrm{N}^{+}$nylon membranes (Millipore, Darmstadt, Germany) in $0.5 \times$ TBE (Tris-borate-EDTA) buffer at $300 \mathrm{~mA}$ at $4^{\circ} \mathrm{C}$ for $30 \mathrm{~min}$. The products were visualized by using enhanced chemiluminesence reagent.

\subsection{Statistical analysis}


Data are expressed in form of mean \pm SD. Differences were compared by ANOVA followed by the Bonferroni correction for a post hoc $t$ test appropriately. Probabilities of $<0.05$ were defined as statistical significance. GraphPad Prism software version 5.0 (GraphPad Software, San Diego, CA, USA) was used to perform all statistical analyses.

\section{Results}

\subsection{Hemodynamic data}

The baseline hemodynamic data and the effects of drugs are shown in (Fig. 2A) and (Fig. 2B). Among the groups, the baseline HR or mean arterial pressures (MAP) showed no statistical difference, with values of $403 \pm 19 \mathrm{bpm}$ and $101 \pm 9 \mathrm{mmHg}$, respectively. HR and MAP in all groups except the sham group were significantly decreased than baseline values after $5 \mathrm{~min}$ of coronary artery occlusion. Compared with the I/R group, both HR and MAP were significantly decreased during application of NECA (in NECA group reperfusion 10min and reperfusion 45min), This effects of NECA on HR and MAP were not affected by pretreatment with ZM241385(in NECA + ZM group) or Go6976 (in NECA + Go group). Compared with the I/R group, CGS treatment also significantly decreased MAP but not HR (in CGS group reperfusion 10min and reperfusion 45min), the PMA (in PMA group) or PMA + Go6976 (in PMA + Go group) treatments had no effect on HR and MAP.

\subsection{NECA treatment attenuated MI/RI by activating A2AR}

We investigated whether NECA had cardioprotective effects against MI/RI in type 2 diabetic rats. NECA, a non-selective but potent adenosine receptor agonist, was infused as a bolus dose of $2 \mu \mathrm{g} / \mathrm{kg}$ before $5 \mathrm{~min}$ of reperfusion, and after application of the bolus, an infusion of $0.2 \mu \mathrm{g} / \mathrm{kg} / \mathrm{min}$ was continued for $65 \mathrm{~min}$ as described in a previous study $[25,26]$. After $2 \mathrm{~h}$ of reperfusion, left ventricular function, left ventricular area at risk (AAR), left ventricular infarct size, plasma cTnl concentration and myocardial apoptosis were examined. As shown in (Fig. 4B), Among the groups, the AAR showed no statistical difference, with values ranging from $28.9 \pm 1.3 \%$ to $31.2 \pm 0.8 \%$; However, in comparison with the I/R group, echocardiography showed an augmentation of LVEF ( $85.1 \pm 2.1 \%$ vs $70.5 \pm 2.7 \%)$ and LVFS $(51.4 \pm 2.5 \%$ vs $37.8 \pm 1.7 \%$ ) in the NECA treated group (Fig. 3), besides, the NECA treatment group also showed reduced infarct size from $58.3 \pm 3.0 \%$ to $31.0 \pm 2.3 \%$ (Fig. 4A, C), plasma cTnl level from $212.0 \pm 11.6$ $\mathrm{pg} / \mathrm{ml}$ to $106.5 \pm 7.6 \mathrm{pg} / \mathrm{ml}$ (Fig. $4 \mathrm{~F}$ ) and apoptotic index from $51.1 \pm 1.4 \%$ to $15.1 \pm 1.1 \%$ (Fig. 4D,E). These results proved that NECA treatment inhibited I/R-induced cardiomyocyte apoptosis and ameliorate cardiac function.

To investigate whether the A2AR is involved in the NECA cardioprotective effects, we administered a selective A2AR antagonist, ZM241385 (0.2 $\mathrm{mg} / \mathrm{kg}$ iv) to the NECA + ZM group before 5 min of NECA as a bolus to ensure proper receptor blockade as previously described [27]. Compared with the NECA group, ZM241385 pretreatment decreased LVEF from $85.1 \pm 2.1 \%$ to $74.0 \pm 2.1 \%$ (Fig. 3), decreased LVFS from $51.4 \pm 2.5 \%$ to $41.9 \pm 1.5 \%$ (Fig. 3), increased infarct size from $31.0 \pm 2.3 \%$ to $51.0 \pm 2.5 \%$ (Fig. $4 \mathrm{~A}, \mathrm{C}$ ), increased plasma cTnl concentration from $106.5 \pm 7.6 \mathrm{pg} / \mathrm{ml}$ to $199.0 \pm 8.8 \mathrm{pg} / \mathrm{ml}$ (Fig. 4F) and increased 
apoptotic index from $15.1 \pm 1.1 \%$ to $36.9 \pm 2.1 \%$ (Fig. 4D, E), These findings indicated that the NECA cardioprotective effects are largely dependent on A2AR.

To further confirmed the protective effects of A2AR, we applied a highly selective A2AR agonist CGS21680 in the CGS group, CGS21680 $30 \mu \mathrm{g} / \mathrm{kg}$ iv 5 min before the onset of reperfusion as a bolus, followed by a $60 \mathrm{~min}$ infusion of $3 \mu \mathrm{g} / \mathrm{kg} / \mathrm{min}$ as previous described[26]. As expected, activation of A2AR specifically alleviated MI/RI. Compared with the I/R group, CGS21680 treatment increased LVEF from $70.5 \pm 2.7 \%$ to $80.9 \pm 3.4 \%$ (Fig. 3), increased LVFS from $37.8 \pm 1.7 \%$ to $48.5 \pm 1.7 \%$ (Fig. 3 ); decreased infarct size from $58.3 \pm 3.0 \%$ to $40.7 \pm 2.8 \%$ (Fig. 4 A, C), decreased plasma cTnl level from $212.0 \pm 11.6$ $\mathrm{pg} / \mathrm{ml}$ to $127.1 \pm 7.7 \mathrm{pg} / \mathrm{ml}$ (Fig. 4F) and decreased the apoptotic index from $51.1 \pm 1.4 \%$ to $19.0 \pm 0.9 \%$ (Fig. 4D, E). These data indicated that A2AR activation is important to protecting the heart against MI/RI in diabetic rats.

\subsection{NECA treatment down-regulates the expression of miR- $15 \mathrm{a}$ and inhibits apoptosis signaling pathway}

Based on recent data showing miRNA-15 family dysregulation during MI/R[16, 17], we examined whether the miRNA-15 family is also involved in the protective effects of NECA. As shown in (Fig. 5A) all members of the miR-15 family (miR-15a, miR-15b, miR-16, miR-195, and miR-497) were significantly upregulated in the infarcted region after MI/R, and NECA treatment markedly inhibited the l/R-induced upregulation of miR-15a but had negligible effects on the other members of the miR-15 family. The effects of NECA on miR-15a were aborted by pretreatment with ZM241385(Fig. 5B). Interestingly, CGS21680 treatment are also inhibited the upregulation of miR-15a as well as NECA (Fig. 5B). These results demonstrated that NECA treatment inhibited I/R-induced miR-15a upregulation by A2AR.

MiR-15a were confirmed to promote cardiomyocyte apoptosis by inhibited the expression of Bcl-2. We next evaluated the effects of NECA on cardiomyocytic apoptosis pathway. The expression levels of Bcl-2, Bax and cleaved-caspase-3 are shown in (Fig. 5C, D, E). The expression of Bcl-2 are decreased and the expression of Bax are increased after MI/R compared with the sham group $(P<0.01)$. NECA treatment inhibited the down-regulation of $\mathrm{Bcl}-2$ and inhibited the upregulation of $\mathrm{Bax}(\mathrm{P}<0.01$ vs the I/R group). And I/R-induced caspase- 3 activation was markedly inhibited by treatment with NECA, and similar effects were observed in the CGS21680 treatment group. In addition, ZM-241385 blocked the effects of NECA on $\mathrm{Bcl} 2$, Bax and Caspase 3 . These results indicated that A2AR is involved in NECA inhibition of the apoptotic signaling pathway. Previous study[16]had demonstrated that miR15a aggravate apoptosis by targeting Bcl-2 mRNA 3'UTR (Fig. 5G). In present study an inverse relationship was noted between miR15 a expression and Bcl-2 protein levels in l/R myocardium (Fig. $5 \mathrm{H}$ ), suggested that miR-15a mediates its proapoptotic effect likely through $\mathrm{Bcl}-2$.

\subsection{NECA treatment increased the phosphorylation and translocation of PKCa}


To investigate the underlying signaling pathways involved in NECA's cardiac protective effects, detection of the phosphorylation levels of PKCa and total PKCa expressions was performed through Western blot analyses and the localization of PKCa isoforms was observed by confocal microscopy. Activation of PKCa by phosphorylation or translocation was measured in I/R myocardium in previously studies[31, 32]. As shown in (Fig. 6), both I/R and drug treatment resulted in increased total PKCa expression, but these changes were not statistically significant (Fig. 6C). However, the phosphorylation of PKCa was enhanced $4.2 \pm 0.7$ fold (Fig. 6B) in the I/R group compared with the sham group. Surprisingly, NECA treatment further increased the phosphorylation levels of PKCa by $14.2 \pm 0.8$ fold ( $P<0.01$ vs the I/R group), and pretreatment with ZM241385 reduced the phosphorylation of PKCa from $14.2 \pm 0.8$ to $5.9 \pm 0.6$ fold $(P<$ 0.01 vs the NECA group). In addition, CGS21680 treatment increased the phosphorylation of PKCa reach to $13.4 \pm 1.1$ fold ( $P<0.01$ vs the I/R group). These findings indicated that NECA activates PKCa through A2AR.

The translocation of PKCa was observed by confocal microscopy. As shown in (Fig. 6D), PKCa displayed a punctate pattern primarily in the cytosol in unstimulated myocardium (sham group) and aggregated in myocardial membranes after MI/R(I/R group). However, PKCa accumulated in a punctate perinuclear pattern after treatment with NECA(in the NECA group).After pretreatment with ZM241385, the translocation of PKCa to the nuclei was decreased (in the NECA + ZM group). In the CGS21680 treatment group, $\mathrm{PKCa}$ also translocated to the perinuclear region (CGS group). These results further indicated that NECA activates PKCa via A2AR.

\subsection{Go6976 abolished the cardioprotective effects of NECA}

Previously, our results indicated that the myocardial protection of NECA by binding with the A2AR was associated with $\mathrm{PKCa}$ signaling activation. For further investigation of the potential mechanisms about the NECA mediated cardioprotective effects, we introduced PMA, a non-selective but potent PKCa activator, and Go6976, a selective PKCa inhibitor, in our experiment.

As depicted in (Fig. 7), Go6976 pretreatment abolished NECA's protective effects. Compared with the NECA treatment, $1 \mu \mathrm{M}$ Go6976 infused in a total of $1 \mathrm{ml}$ over $10 \mathrm{~min}$ before $5 \mathrm{~min}$ of NECA increased the infarct size from $31.0 \pm 2.3 \% \%$ to $54.7 \pm 1.7 \%$ (Fig. 7D), the plasma cTnl level from $106.5 \pm 7.6 \mathrm{pg} / \mathrm{ml}$ to $189.5 \pm 9.0 \mathrm{pg} / \mathrm{ml}$ (Fig. 7E) and the apoptotic index from $15.1 \pm 1.1 \%$ to $36.2 \pm 1.6 \%$ (Fig. 7F). These findings suggested that the NECA mediated anti-post-MI/R apoptosis effects are dependent on PKCa activation.

To confirmed the protective effects of PKCa, PMA were used in our experiment. As shown in (Fig. 7), PMA treatment significantly reduced infarct size from $58.3 \pm 3.0 \%$ to $37.5 \pm 2.0 \%$, cTnl release from $212.0 \pm$ $11.6 \mathrm{pg} / \mathrm{ml}$ to $118.6 \pm 7.3 \mathrm{pg} / \mathrm{ml}$ and the apoptotic index from $51.1 \pm 1.4 \%$ to $16.2 \pm 1.0 \%$ compared with those of the I/R group $(P<0.01)$, and these effects were abolished by co-treatment with Go6976.which further indicated PKCa activation have potent cardioprotective effects. 
We further explored the role of PKCa in the myocardial apoptosis signaling pathway. As shown by previous observations, NECA's cardioprotection was associated with PKCa activation, and thus, we next determined whether administration of Go6976 abolished the NECA mediated anti-apoptotic effect, As shown in (Fig. 7G-J), compared with NECA treatment, Go6976 pretreatment consolidated apoptosis signaling by reduce Bcl-2 $(P<0.01)$ while augmenting Bax and Caspase-3 expression $(P<0.01)$. Furthermore, activation of PKCa by PMA were also increased the expression of $\mathrm{Bcl}-2$ and decreased the expression of Bax and Cleaved-caspase3, these beneficial effects were aborted by pretreatment with Go6976. These data suggested that NECA reduced myocardial apoptosis dependent on PKCa signaling pathway.

\subsection{Go6976 abolished the inhibition of miR-15a by NECA}

Previously, we demonstrated that NECA inhibited the upregulation of miR-15a after MI/R. We further studied the effect of Go6976 on NECA 's inhibition. As shown in (Fig. 8A), NECA-induced miR-15a downregulation was abolished by pretreatment with Go6976, suggesting that the effect of NECA on miR$15 a$ is dependent on PKCa. PMA treatment also inhibit l/R-induced miR-15a upregulation, which was blocked by pretreatment with Go6976; this finding demonstrated that miR-15a was manipulated by PKCa.

To further investigate how PKCa inhibits miR-15a expression in I/R-injured myocardium, we performed an EMSA using nuclear proteins from the infarct zone and a biotin-labeled oligonucleotide representing the sequence of pri-miRNA-15a that contain the PKCa binding site (GATTTTGAA). As shown in (Fig. 8B), the biotin-labeled pri-miR-15a probe formed a RNA-protein complex with PKCa. The complex band disappeared with addition of 200x unlabeled ("cold") competitor but re-appeared when mutated unlabeled probe was added. Supershift analysis with an antibody specific for $\mathrm{PKCa}$, identified this isoform as a component of the observed complex. The results indicated that PKCa can bind directly to pri-miR-15a. Therefore, our data confirmed that PKCa inhibited miR-15a expression by binding directly to pri-miRNA-15a in diabetic rat hearts after MI/R.

\section{Discussion}

Myocardial infarction is one of the most important causes of mortality worldwide. Timely recovery myocardial perfusion remains the major treatment for acute $\mathrm{MI}$ to salvage the ischemic myocardium, but reperfusion result in the excess formation of reactive oxygen species, mitochondrial dysfunction, intracellular calcium overload, uncoordinated excess contractile activity and activation of intracellular proteolysis caused irreversible myocardial injury [33, 34]. IPO, a phenomenon involving brief repetitive episodes of I/R at the directed onset of reperfusion, can effectively limit MI/RI in both experimental and clinical situations[35]. Endogenous adenosine release to activate adenosine receptors during reperfusion plays a key role in the IPO-mediating cardioprotective effect. Subsequently, abundant experimental and clinical studies have demonstrated that adenosine applied directly before the onset of reperfusion also alleviates I/R-induced myocardial injury[34]. However, numerous studies have reported that the cardioprotective effect of IPO is considerably suppressed in chronic diabetic myocardium due to the development of metabolic abnormalities[9] And T2MD is associated with enhanced uptake of interstitial 
adenosine by cardiac fibroblasts and reduced ability of these cells to release adenosine during ATP deprivation[36].

Previous studies have confirmed that adenosine limits infarct size only with massive adenosine receptor activation by a prolonged high-dose infusion of adenosine but not a short-acting bolus injection[5, 34]. In the present study, we increased extracellular adenosine concentrations in diabetic hearts during reperfusion by continuous intravenous administration of NECA. a non-selective adenosine receptor agonist ( $\mathrm{Ki}=14 \mathrm{nM}, 20 \mathrm{nM}$, and $6.2 \mathrm{nM}$ for human A1R, A2AR, and A3R, respectively, and $E C 50=2,400$ $\mathrm{nM}$ for A2BR[5]). Our study showed for the first time that administration of NECA $(2 \mu \mathrm{g} / \mathrm{kg})$ infused 5 min before the onset of reperfusion followed by $0.2 \mu \mathrm{g} / \mathrm{kg} / \mathrm{min}$ infusion for $60 \mathrm{~min}$ exerted a cardioprotective effect in vivo in T2MD rat hearts, as demonstrated by the improved post-MI/R cardiac functional recovery, reduced myocardial infarct size and cTnl release and attenuated myocardial apoptosis (Fig. 3-5). These findings indicated that maintaining high extracellular adenosine concentrations in cardiac tissue can alleviate $\mathrm{MI} / \mathrm{RI}$ even in the diabetic state. A similar phenomenon was observed in non-diabetic rabbits and mice[25, 26].

Adenosine exerts cardioprotective effects by binding to any of four $G$ protein-coupled receptors $(A 1 R$, $A 2 A R, A 2 B R$ and A3R) $[5,6]$, and abundant studies have revealed that A1R and A3R agonists protect cardiomyocytes against ischemic injury, while A2AR and A2BR agonists function primarily by attenuating reperfusion injury to the heart $[5,11,37,38]$. However, $A 2 B R$ is a low-affinity receptor that requires high adenosine concentrations to be significantly activated, and normal adenosine concentrations fail to activate this receptor $[6,34]$. Therefore, NECA postconditioning alleviate I/R-induced myocardial injury predominantly by activating A2AR[39, 40]. Our study found that the anti-MI/RI effect of NECA was reversed by the selective A2A antagonist ZM241385(222-fold more potent for A2A than A2B) and mimicked by CGS21680, a selective A2A agonist ( $\mathrm{Ki}=290 \mathrm{nM}, 27 \mathrm{nM}$, and $67 \mathrm{nM}$ for A1R, A2AR, and A3R, $\mathrm{EC50}=88,800 \mathrm{nM}$ for A2BR) (Fig. 3-5) suggested that A2AR are primarily involved in the action of NECA during reperfusion. Similarly, the anti-infarction effect of NECA was abolished by the selective A2AR antagonist SCH58261 in vitro in a non-diabetic rat heart[38].

Previous studies have demonstrated that adenosine can increase PKC activity by binding with adenosine receptors $[5,41]$, and activation of PKC was also confirmed to protect the myocardium against I/R. Therefore, it is logical that adenosine alleviates MI/RI by activating PKC, but the underlying mechanisms and the specific PKC isoforms involved in this protective cascade are unclear and have been widely investigated. PKC isozymes as a family of serine/threonine kinases including classical PKCs $(\alpha, \beta 1, \beta 2$, $\gamma)$, novel PKCs $(\delta, \varepsilon, \eta, \theta)$, and atypical PKCs $(\zeta, \lambda)$, were confirmed to be expressed in cardiac tissues from various mammalian species including mice, rats, rabbits, dogs and pigs[10]. phosphorylation or translocation is the activation state of PKC isoforms[31]. Wang et al. found that PKCa, PKCס and PKC $\varepsilon$ were translocated from the soluble to the particulate fraction in response to $H / R(120 \mathrm{~min} / 30 \mathrm{~min})$ in rat primary cultured cardiomyocytes, and inhibition of the translocation of PKCa reduced I/R-induced apoptosis and myocardial injury. Moreover, Hsu et al.[42]reported that decreased $\mathrm{PKCa} / \varepsilon$ activity (reduction in the phosphorylation of $\mathrm{PKCa} / \varepsilon$ by Go6976/عV1-2) also provided cardioprotection against 
I/R-induced heart injury. However, Zatta et al.[43]reported that IPO limited infarct size by increasing the translocation of PKC $\varepsilon$ to sites outside the mitochondrial outer membrane but limited translocation of PKC $\delta$ to the mitochondria. Additionally, Lu et al.[30] showed that IPC increasing the translocation of PKCa and PKCS from the cytosol to the sarcolemma in rat heart, if inhibited the translocation of PKCa will abolish cardioprotection induced by IPC[30, 44]. Furthermore, PKCa was shown to be activated in the heart and associated with sevoflurane-, fibroblast growth factor-2- and sildenafil-induced cardioprotection[2, 31]. Therefore, different PKC isoforms may play opposite roles in regulating MI/RI.

In our experimental model, we found that NECA treatment significantly increased the phosphorylation level of PKCa, and this effect was aborted by ZM241385 and mimicked by CGS21680 (Fig. 6). NECA treatment also resulted in PKCa translocation from the cytosol to the nucleus but not the membranes, and this phenomenon disappeared following pretreatment with ZM241385 and reappeared following administration of CGS21680 alone (Fig. 6). These findings indicated that NECA-induced activation of PKCa was dependent on A2AR. In addition, the cardioprotection of NECA was ablated by the PKCa selective inhibitor Go6976. PMA postconditioning also ameliorated MI/RI, as shown by the reduced infarct size, apoptotic index and cTnl release (Fig. 7A-F). These beneficial effects were weakened by coadministration of Go6976. The data in the present study demonstrated that NECA-induced cardioprotection depends on the activation of $\mathrm{PKCa}$, which play essential roles in this process. However, different adenosine receptor subtypes corresponding to specific PKC isoforms may be involved. Mitsuhiro et al[45] reported that the A1R mediated cardioprotection via activation of the PKC $\delta$ signaling pathway, and $A 1 R s$ were also reported to promote the translocation of PKC $\square$ and PKC $\delta$ to the plasma membrane in rat cardio myocytes[46]. However, the A3Rs have an opposite role in PKC activation, A3R agonist IB-MECA was shown to attenuate sunitinib-induced PKCa phosphorylate[47]; and A2BR was described as a downstream signal in the PKCs signaling pathway[25, 48]. Our data suggest that NECA can activate PKCa via A2AR.

Next, we investigated how NECA-induced PKC-a activation contributes to attenuation MI/RI. Increasing evidence suggested that apoptosis leads to the reduction of viable contractile cardiomyocytes[28]. Persistent acute ischemia can trigger apoptosis, reperfusion boosts this process, diabetic metabolic abnormalities further aggravation[49]. Suppressing myocardial apoptosis could reduce the loss of contractile cells attenuated cardiac injury and improve cardiac function[28, 49]. Numerous studies have shown that the miR-15 family has an effect on I/R-induced cardiomyocyte apoptosis. The expression of the miR-15 family was upregulated in the infarcted cardiac region including miR-15a, miR-15b, miR-16, miR-195, and miR-497 after Ml, which increases cardiomyocyte apoptosis by negatively regulating the expression of target genes[16]. miR-15a, a member of miR-15 family was shown to aggravate myocardial injury by promote cardiomyocyte apoptosis via targeting Bcl-2. Because miR-15a family members have the same 'seed sequence' for mRNA recognition (Fig. 5G), they are expected to show an overlap in targets. miR-15b, miR-16,miR-195 and miR-497 were also confirmed to promote cardiomyocyte apoptosis and exacerbate cardiac injury by targeting Bcl-2, and downregulation of miR-15family members recovered Bcl2 protein expression and attenuated I/R-induced apoptosis[18, 19]. 
Interestingly, PKCa has been demonstrated to promote cell survival and suppress apoptosis by increasing the activity of the anti-apoptotic protein Bcl-2[2]. Furthermore, Cohen et al.[20]showed that upregulated $\mathrm{PKCa}$ is inversely related to decreased production of miR-15a in head and neck squamous cell carcinoma, and similarly Brandenstein et al. showed that PKCa inhibited apoptosis and promoted cell proliferation by binding directly to pri-miRNA-15a in the nucleus, which reduced mature cytoplasmic miR-15a levels[21, 22]. Therefore, we concluded that PKCa regulate Bcl-2 activity by inhibiting the expression of miR-15a in I/R hearts. In the present study we found that NECA, CGS and PMA-induced PKCa activation is associated with down-regulation miR-15a expression and upregulation Bcl-2 protein level. Furthermore, we confirmed that PKCa binding directly to pri-miRNA-15a in the nucleus by EMSA (Fig. 8B), which confirmed our hypothesis that PKCa regulates $\mathrm{Bcl}-2$ activity by inhibiting the expression of miR-15a.

In summary, our findings suggested that NECA reduces $\mathrm{MI} / \mathrm{RI}$ and cardiomyocytes apoptosis in T2DM rats through the A2AR/PKCa/miR-15a signal pathway (illustrated in Fig. 9). Our study suggested that NECA is a useful target candidate for the treatment of MI/RI in patient with type 2 diabetes.

\section{Declarations}

Acknowledgements We thank Experimental Animal Center of Zhongnan Hospital for providing its SPFclass animal laboratory

Author contributions All authors listed have made substantial, direct, and intellectual contribution to the work and approved it for publication.

Funding Information This work was supported by the National Natural Science Foundation of China $(81471858,81871553)$ and the Science and Technology Fund of Guizhou Provincial Health Commission (grant number gzwkj2021-274)

Data Availability Statement The data will be made available by the corresponding author upon request.

Conflict of interest The authors declare that they have no conflicts of interest

Ethical Approval All animal experimental protocols were approved by the Institutional Ethics Committee of Zhongnan Hospital of Wuhan University . And all procedures were carried out according to the guide for the Care and US National Institute of Health (NIH Publication No. 85-23, revised 1996).

\section{References}

1. Li H, Yao W, Liu Z, Xu A, Huang Y, Ma XL, et al. Hyperglycemia Abrogates Ischemic Postconditioning Cardioprotection by Impairing AdipoR1/Caveolin-3/STAT3 Signaling in Diabetic Rats. DIABETES. 2016;65:942-55.

2. Bouwman RA, Musters RJ, van Beek-Harmsen BJ, de Lange JJ, Lamberts RR, Loer SA, et al. Sevoflurane-induced cardioprotection depends on PKC-alpha activation via production of reactive 
oxygen species. Br J Anaesth. 2007;99:639-45.

3. Buddeke J, Bots ML, van Dis I, Visseren FL, Hollander M, Schellevis FG, et al. Comorbidity in patients with cardiovascular disease in primary care: a cohort study with routine healthcare data. Br J Gen Pract. 2019;69:e398-406.

4. Das A, Salloum FN, Filippone SM, Durrant DE, Rokosh G, Bolli R, et al. Inhibition of mammalian target of rapamycin protects against reperfusion injury in diabetic heart through STAT3 signaling. BASIC RES CARDIOL. 2015;110:31.

5. Cohen MV, Downey JM. Adenosine: trigger and mediator of cardioprotection. BASIC RES CARDIOL. 2008;103:203-15.

6. Mclntosh VJ, Lasley RD. Adenosine Receptor-Mediated Cardioprotection: Are All 4 Subtypes Required or Redundant? J CARDIOVASC PHARM T. 2012;17:21-33.

7. Feliu C, Peyret H, Poitevin G, Cazaubon Y, Oszust F, Nguyen P, et al. Complementary Role of P2 and Adenosine Receptors in ATP Induced-Anti-Apoptotic Effects Against Hypoxic Injury of HUVECs. INT J MOL SCI 2019;20.

8. Headrick JP, Ashton KJ, Rose'Meyer RB, Peart JN. Cardiovascular adenosine receptors: expression, actions and interactions. Pharmacol Ther. 2013;140:92-111.

9. Penna C, Andreadou I, Aragno M, Beauloye C, Bertrand L, Lazou A, et al. Effect of hyperglycaemia and diabetes on acute myocardial ischaemia-reperfusion injury and cardioprotection by ischaemic conditioning protocols. Br J Pharmacol. 2020;177:5312-35.

10. Singh RM, Cummings E, Pantos C, Singh J. Protein kinase C and cardiac dysfunction: a review. HEART FAIL REV. 2017;22:843-59.

11. Cohen MV, Downey JM. Signalling pathways and mechanisms of protection in pre- and postconditioning: historical perspective and lessons for the future. Br J Pharmacol. 2015;172:191332.

12. Ren X, Roessler AE, Lynch TT, Haar L, Mallick F, Lui Y, et al. Cardioprotection via the skin: nociceptorinduced conditioning against cardiac MI in the NIC of time. Am J Physiol Heart Circ Physiol. 2019;316:H543-53.

13. Wu G, Wang Z, Shan P, Huang S, Lin S, Huang W, et al. Suppression of Netrin-1 attenuates angiotension II-induced cardiac remodeling through the PKC/MAPK signaling pathway. BIOMED PHARMACOTHER. 2020;130:110495.

14. Tsai CY, Wen SY, Cheng SY, Wang CH, Yang YC, Viswanadha VP, et al. Nrf2 Activation as a Protective Feedback to Limit Cell Death in High Glucose-Exposed Cardiomyocytes. J CELL BIOCHEM. 2017;118:1659-69.

15. Diez ER, Sanchez JA, Prado NJ, Ponce ZA, Garcia-Dorado D, Miatello RM, et al. Ischemic Postconditioning Reduces Reperfusion Arrhythmias by Adenosine Receptors and Protein Kinase C Activation but Is Independent of KATP Channels or Connexin 43. INT J MOL SCI 2019;20.

16. Hullinger TG, Montgomery RL, Seto AG, Dickinson BA, Semus HM, Lynch JM, et al. Inhibition of miR15 Protects Against Cardiac Ischemic InjuryNovelty and Significance. CIRC RES. 2012;110:71-81. 
17. Braga L, Ali H, Secco I, Giacca M. Non-coding RNA therapeutics for cardiac regeneration. CARDIOVASC RES. 2021;117:674-93.

18. Liu L, Zhang G, Liang Z, Liu X, Li T, Fan J, et al. MicroRNA-15b enhances hypoxia/reoxygenationinduced apoptosis of cardiomyocytes via a mitochondrial apoptotic pathway. APOPTOSIS. 2014;19:19-29.

19. Liu LF, Liang Z, Lv ZR, Liu XH, Bai J, Chen J, et al. MicroRNA-15a/b are up-regulated in response to myocardial ischemia/reperfusion injury. J GERIATR CARDIOL. 2012;9:28-32.

20. Cohen EE, Zhu H, Lingen MW, Martin LE, Kuo WL, Choi EA, et al. A feed-forward loop involving protein kinase Calpha and microRNAs regulates tumor cell cycle. CANCER RES. 2009;69:65-74.

21. von Brandenstein $M$, Depping $R$, Schäfer $E$, Dienes $H$, Fries JWU. Protein kinase $C$ a regulates nuclear pri-microRNA 15a release as part of endothelin signaling. Biochimica et Biophysica Acta (BBA) Molecular Cell Research. 2011;1813:1793-802.

22. von Brandenstein M, Pandarakalam JJ, Kroon L, Loeser H, Herden J, Braun G, et al. MicroRNA 15a, inversely correlated to PKCalpha, is a potential marker to differentiate between benign and malignant renal tumors in biopsy and urine samples. AM J PATHOL. 2012;180:1787-97.

23. Srinivasan K, Viswanad B, Asrat L, Kaul CL, Ramarao P. Combination of high-fat diet-fed and lowdose streptozotocin-treated rat: A model for type 2 diabetes and pharmacological screening. PHARMACOL RES. 2005;52:313-20.

24. Ke J, Yao B, Li T, Cui S, Ding H. A2 Adenosine Receptor-mediated Cardioprotection Against Reperfusion Injury in Rat Hearts Is Associated With Autophagy Downregulation. J Cardiovasc Pharmacol. 2015;66:25-34.

25. Philipp S, Yang XM, Cui L, Davis AM, Downey JM, Cohen MV. Postconditioning protects rabbit hearts through a protein kinase C-adenosine A2b receptor cascade. CARDIOVASC RES. 2006;70:308-14.

26. Methner C, Schmidt K, Cohen MV, Downey JM, Krieg T. Both A2a and A2b adenosine receptors at reperfusion are necessary to reduce infarct size in mouse hearts. AJP: Heart Circulatory Physiology. 2010;299:H1262-4.

27. Kin H, Zatta AJ, Lofye MT, Amerson BS, Halkos ME, Kerendi F, et al. Postconditioning reduces infarct size via adenosine receptor activation by endogenous adenosine. CARDIOVASC RES. 2005;67:12433.

28. Yu L, Li F, Zhao G, Yang Y, Jin Z, Zhai M, et al. Protective effect of berberine against myocardial ischemia reperfusion injury: role of Notch1/Hes1-PTEN/Akt signaling. APOPTOSIS. 2015;20:796810.

29. Yu L, Liang H, Dong X, Zhao G, Jin Z, Zhai M, et al. Reduced silent information regulator 1 signaling exacerbates myocardial ischemia-reperfusion injury in type 2 diabetic rats and the protective effect of melatonin. J PINEAL RES. 2015;59:376-90.

30. Lu MJ, Chen YS, Huang HS, Ma MC. Hypoxic preconditioning protects rat hearts against ischemiareperfusion injury via the arachidonate12-lipoxygenase/transient receptor potential vanilloid 1 pathway. BASIC RES CARDIOL. 2014;109:414. 
31. Manning JR, Perkins So, Sinclair EA, Gao X, Zhang Y, Newman G, et al. Low molecular weight fibroblast growth factor-2 signals via protein kinase $C$ and myofibrillar proteins to protect against postischemic cardiac dysfunction. Am J Physiol Heart Circ Physiol. 2013;304:H1382-96.

32. Sims MW, Winter J, Brennan S, Norman RI, Ng GA, Squire IB, et al. PKC-mediated toxicity of elevated glucose concentration on cardiomyocyte function. Am J Physiol Heart Circ Physiol. 2014;307:H58797.

33. Hausenloy DJ, Yellon DM. Time to take myocardial reperfusion injury seriously. N Engl J Med. 2008;359:518-20.

34. Yetgin T, Uitterdijk A, Te LHM, Merkus D, Krabbendam-Peters I, van Beusekom HM, et al. Limitation of Infarct Size and No-Reflow by Intracoronary Adenosine Depends Critically on Dose and Duration. JACC Cardiovasc Interv. 2015;8:1990-9.

35. Heusch G. Molecular basis of cardioprotection: signal transduction in ischemic pre-, post-, and remote conditioning. CIRC RES. 2015;116:674-99.

36. Podgorska M, Kocbuch K, Grden M, Szutowicz A, Pawelczyk T. Reduced ability to release adenosine by diabetic rat cardiac fibroblasts due to altered expression of nucleoside transporters. J Physiol. 2006;576:179-89.

37. Laubach VE, French BA, Okusa MD. Targeting of adenosine receptors in ischemia-reperfusion injury. EXPERT OPIN THER TAR. 2010;15:103-18.

38. Xi J, Mclntosh R, Shen X, Lee S, Chanoit G, Criswell H, et al. Adenosine A2A and A2B receptors work in concert to induce a strong protection against reperfusion injury in rat hearts. $J$ MOL CELL CARDIOL. 2009;47:684-90.

39. Patel RA, Glover DK, Broisat A, Kabul HK, Ruiz M, Goodman NC, et al. Reduction in myocardial infarct size at 48 hours after brief intravenous infusion of ATL-146e, a highly selective adenosine A2A receptor agonist. Am J Physiol Heart Circ Physiol. 2009;297:H637-42.

40. Urmaliya VB, Pouton CW, Ledent C, Short JL, White PJ. Cooperative cardioprotection through adenosine $A 1$ and $A 2 A$ receptor agonism in ischemia-reperfused isolated mouse heart. $\mathrm{J}$ Cardiovasc Pharmacol. 2010;56:379-88.

41. Lee JE, Bokoch G, Liang BT. A novel cardioprotective role of RhoA: new signaling mechanism for adenosine. FASEB J. 2001;15:1886-94.

42. Hsu KL, Fan HJ, Chen YC, Huang YS, Chen $\mathrm{CH}$, Wu JC, et al. Protein kinase C-Fyn kinase cascade mediates the oleic acid-induced disassembly of neonatal rat cardiomyocyte adherens junctions. Int $\mathrm{J}$ Biochem Cell Biol. 2009;41:1536-46.

43. ZATTA A, KIN H, LEE G, WANG N, JIANG R, LUST R, et al. Infarct-sparing effect of myocardial postconditioning is dependent on protein kinase $C$ signalling. CARDIOVASC RES. 2006;70:315-24.

44. Hassouna A, Matata BM, Galinanes M. PKC-epsilon is upstream and PKC-alpha is downstream of mitoKATP channels in the signal transduction pathway of ischemic preconditioning of human myocardium. Am J Physiol Cell Physiol. 2004;287:C1418-25. 
45. Kudo M, Wang Y, Xu M, Ayub A, Ashraf M. Adenosine. A(1) receptor mediates late preconditioning via activation of PKC-delta signaling pathway. Am J Physiol Heart Circ Physiol. 2002;283:H296-301.

46. Yang Z, Sun W, Hu K. Adenosine $A(1)$ receptors selectively target protein kinase $C$ isoforms to the caveolin-rich plasma membrane in cardiac myocytes. Biochim Biophys Acta. 2009;1793:1868-75.

47. Sandhu H, Cooper S, Hussain A, Mee C, Maddock H. Attenuation of Sunitinib-induced cardiotoxicity through the A3 adenosine receptor activation. EUR J PHARMACOL. 2017;814:95-105.

48. Kuno A, Critz SD, Cui L, Solodushko V, Yang X, Krahn T, et al. Protein kinase C protects preconditioned rabbit hearts by increasing sensitivity of adenosine $A 2 b$-dependent signaling during early reperfusion. J MOL CELL CARDIOL. 2007;43:262-71.

49. Fu F, Tian F, Zhou H, Lv W, Tie R, Ji L, et al. Semen cassiae attenuates myocardial ischemia and reperfusion injury in high-fat diet streptozotocin-induced type 2 diabetic rats. Am J Chin Med. 2014;42:95-108.

\section{Figures}

\section{Sham group}

30-min regional Ischemia

2-h reperfusion

I/R group

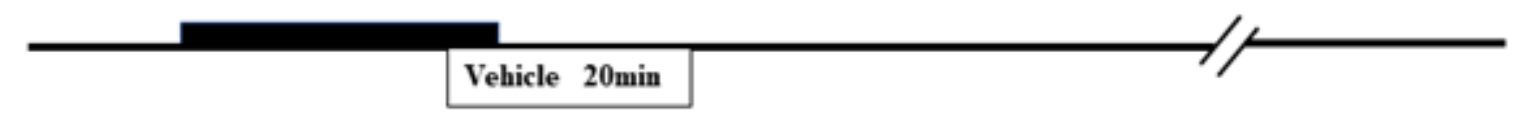

NECA group

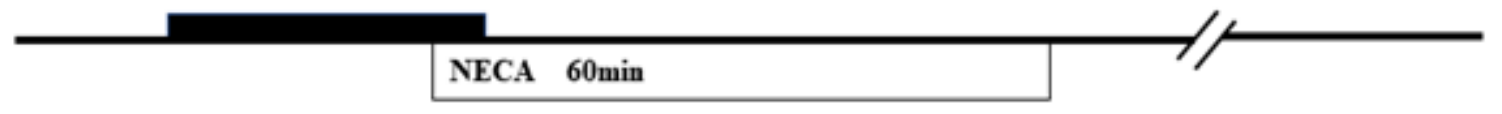

NECA + ZM group

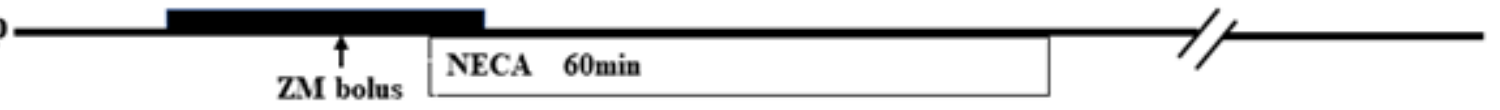

CGS group

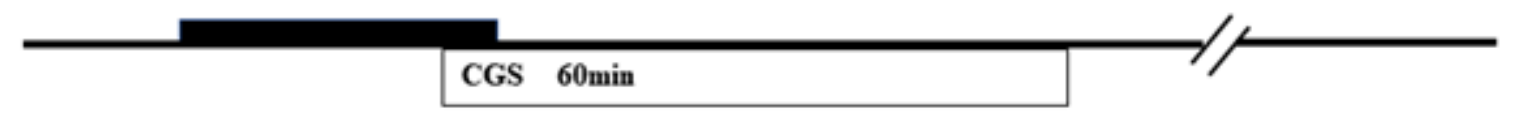

NECA+Go group

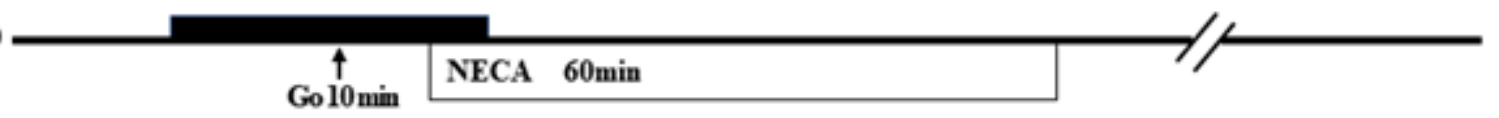

PMA group

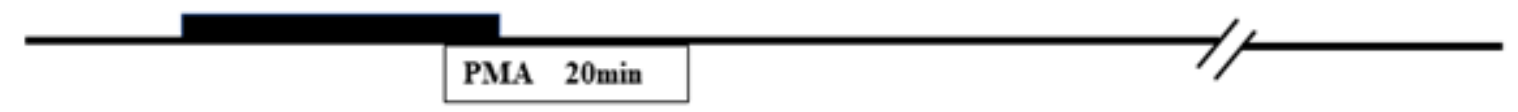

PMA+Go group
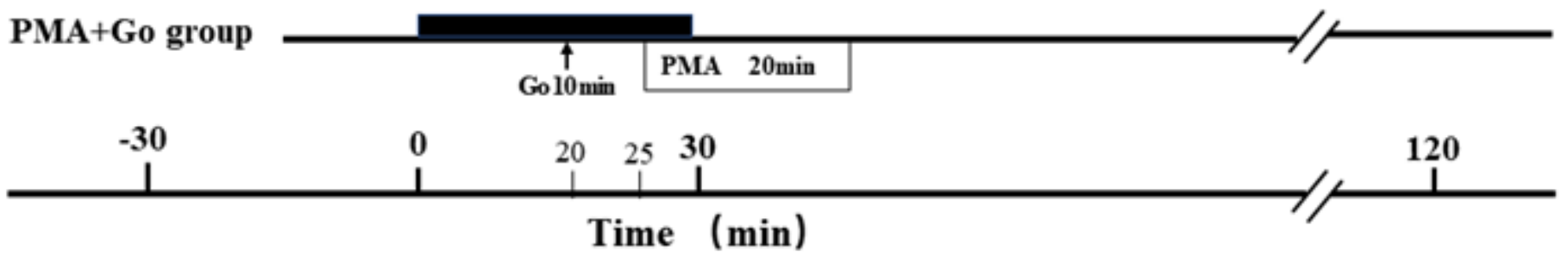

Figure 1 

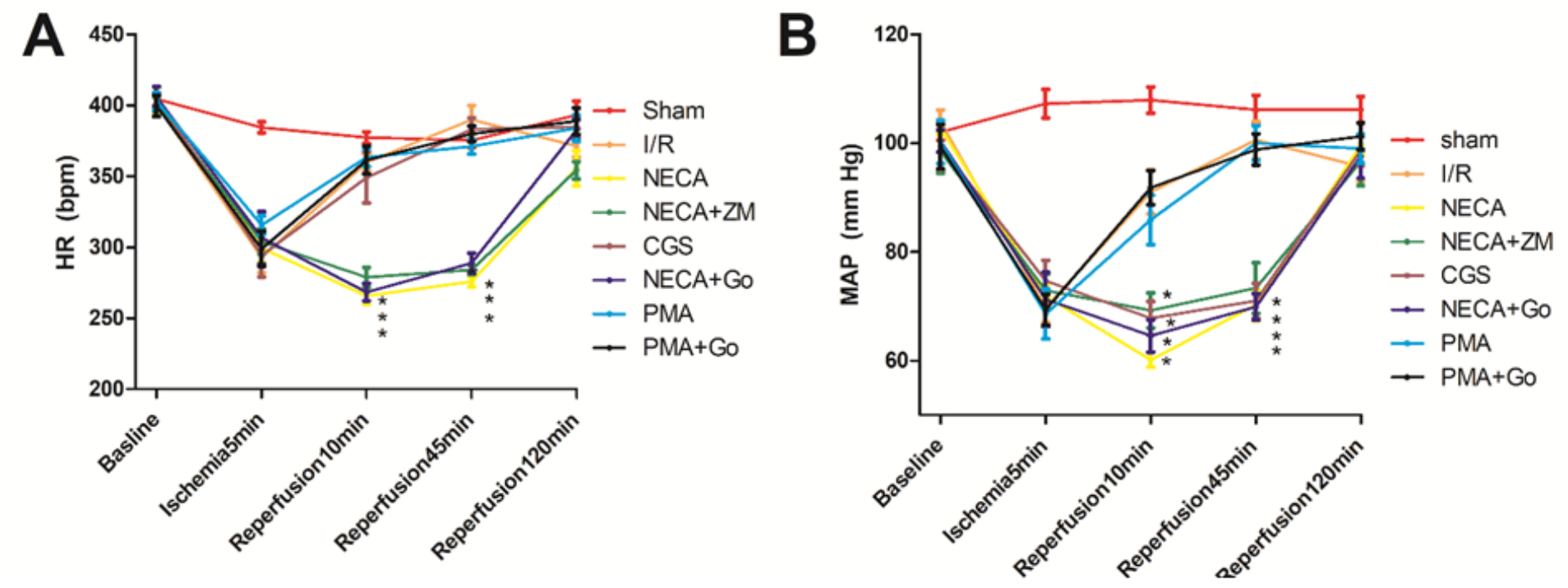

Figure 2

Effects of the drugs on the heart rate (HR) (A) and mean arterial blood pressure (MAP) (B). Data are expressed as mean $\pm S D, n=15$. ${ }^{*} P<0.05$ versus the I/R group.
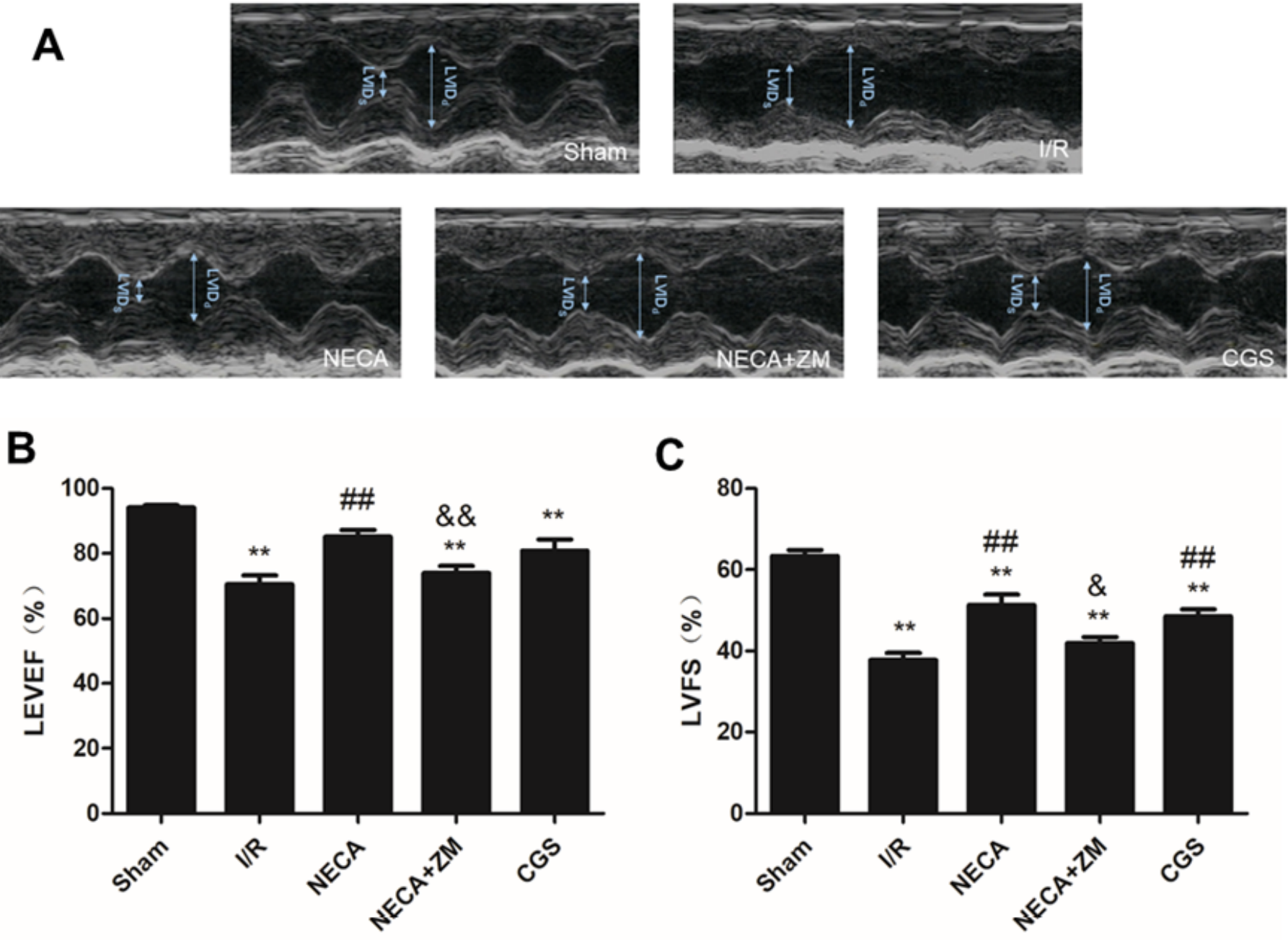


\section{Figure 3}

The effects of the NECA, NECA+ZM and CGS treatments on post-MI/R left ventricular function. (A) Representative motion (M)-mode images by echocardiography. Cardiac function was assessed by echocardiography $2 \mathrm{~h}$ after reperfusion, $\mathrm{HR}$ is between $350 \sim 380 \mathrm{bpm}$. (B) Left ventricular ejection fraction (LVEF). (C) Left ventricular fractional shortening (LVFS). Data are expressed as mean $\pm S D, n=6$. $\star \star P<0.01$ versus the sham group, \#\#P<0.01 versus the I/R group, \&\&P<0.01 versus the NECA group, \&P< 0.05 versus the NECA group.
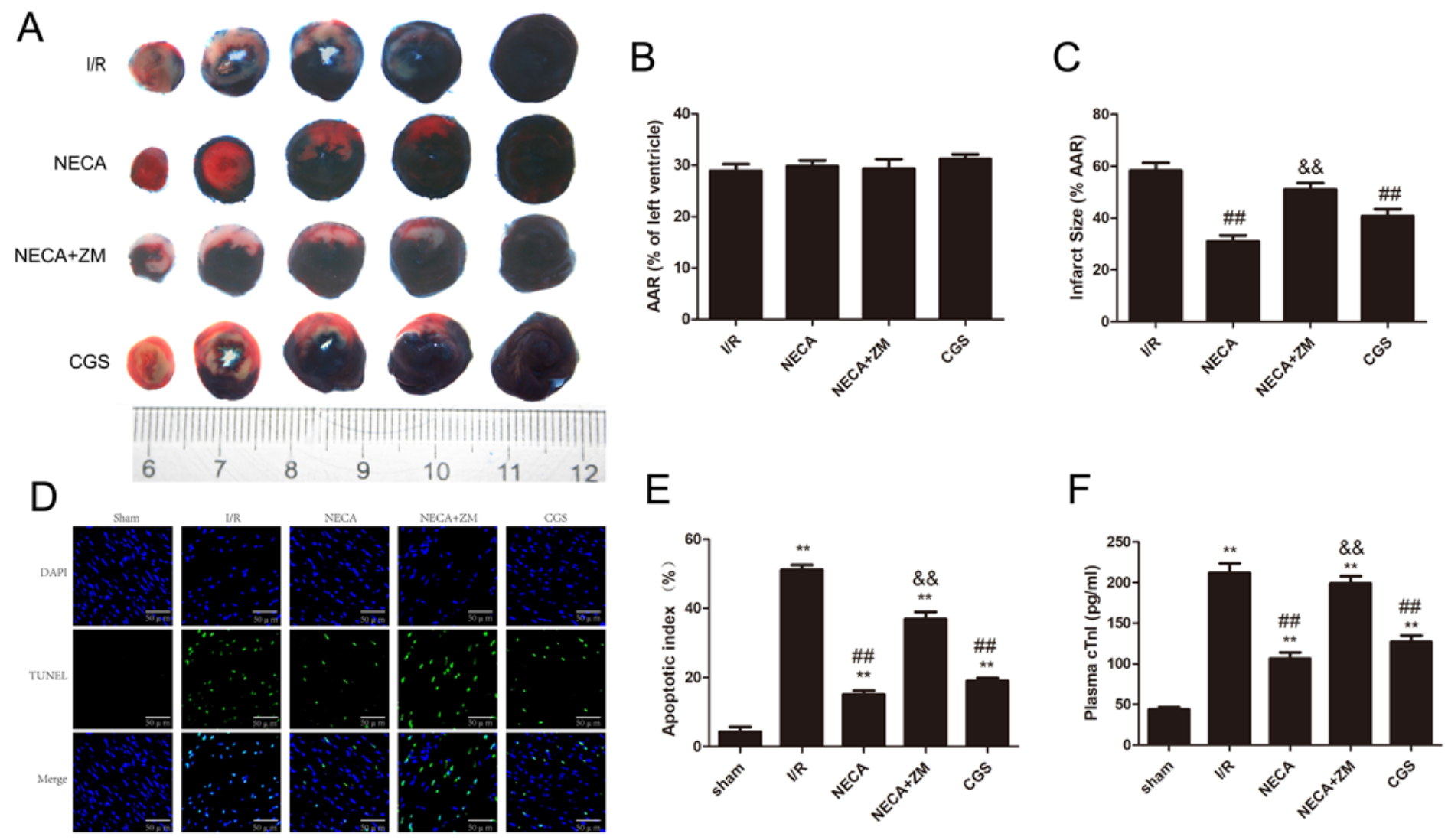

\section{Figure 4}

The effects of the NECA, NECA+ZM and CGS treatments on AAR, infarct size, apoptotic index and Plasma cTnl level. (A) Representative image of cardiac slices determined by TTC and Evans blue staining, the blue-stained area represents the non-ischemic region; red-stained area represents ischemic but not infarcted portion; and the negative-stained area represents the infarcted portion. (B) AAR expressed as a percentage of the total left ventricle. (C) Infarct size expressed as a percentage of the AAR. (D) Representative photomicrographs of in situ detection of apoptotic cardiomyocytes by TUNEL staining. Blue fluorescence indicates nuclei of total cardiomyocytes; green fluorescence indicates TUNEL-positive nuclei; original 200x. (E) Percentage of TUNEL-positive nuclei. (F) Plasma cTnI concentration. Data are expressed as mean $\pm S D, n=9$. ${ }^{*} P<0.01$ versus the sham group, $\# \# P<0.01$ versus the $1 / R$ group, \&\&P< 0.01 versus the NECA group. 
A

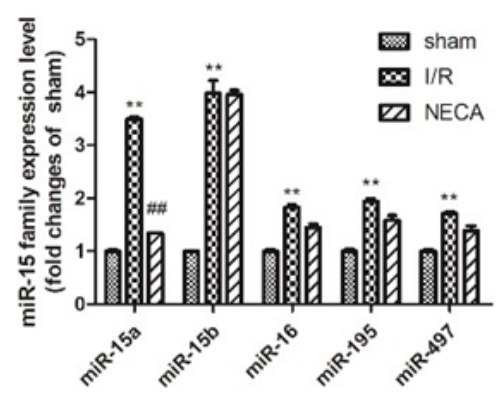

D

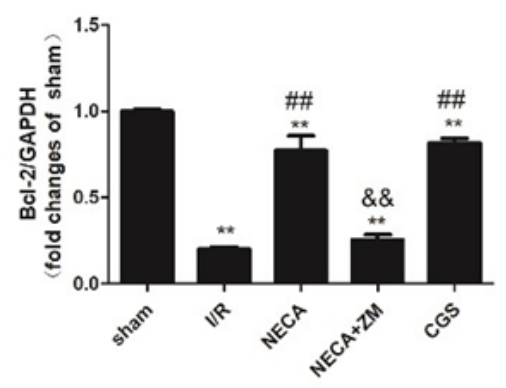

G

Predicted binding sites between miR-15a and 3'UTR of Bcl2

Human BCl2 NM_000633 3' UTR length: 5721

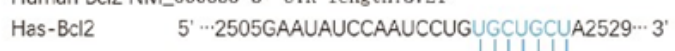

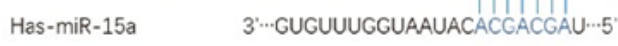

Mouse Bc12 NM 000633 3' UTR length:5077

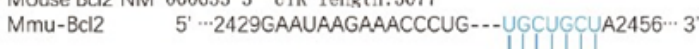

Mmu-miR-15a 3'..'GUGUUUGGUAAUACACGACGAU $\cdots 5^{\prime}$

Rat Bcl2 NM_000633 3' UTR length:4951

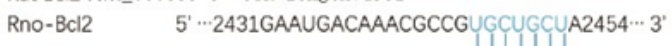

Rno-miR-15a

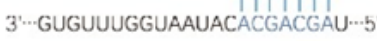

E
C

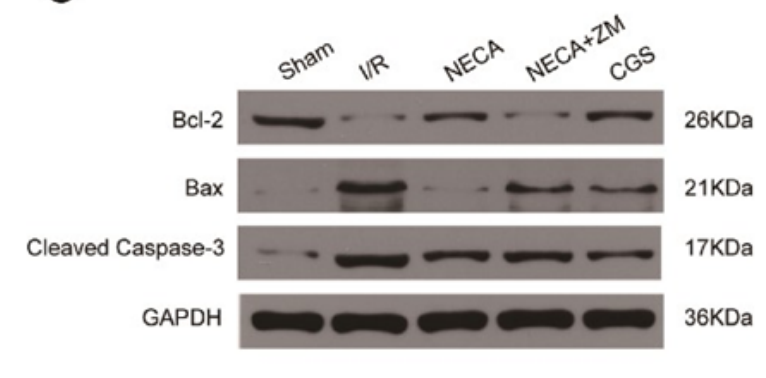

$\mathrm{F}$

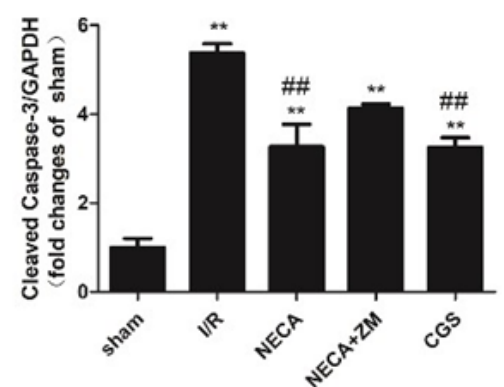

$\mathrm{H}$

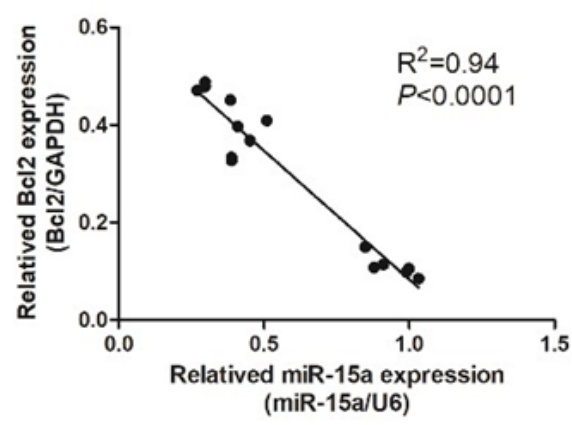

\section{Figure 5}

The effects of the NECA, NECA+ZM and CGS treatments on the miR-15 family members and apoptotic signaling. (A) Real-time qPCR analysis of miR-15 family members expression in the infarct zone. (B) Expression of miR-15a in the different drug treatment groups. (C) Representative immunoblots of Bcl-2, Bax, Cleaved Caspase-3 and GAPDH in the different drug treatment groups. (D) Bcl-2 expression. (E) Bax expression. (F) Cleaved Caspase-3 expression. Data are expressed as means $\pm S E M, n=6 . * * P<0.01$ versus the sham group, \#\#P< 0.01 versus the I/R group, \&\&P< 0.01 versus the NECA group. (G) Conserved miR $15 \mathrm{a}$ binding site in the 3 ' UTR of $\mathrm{Bcl} 2$. $(\mathrm{H})$ The correlation between miR-15a and $\mathrm{Bc} / 2$ protein levels were plotted and analyzed by linear regression analysis. 
A

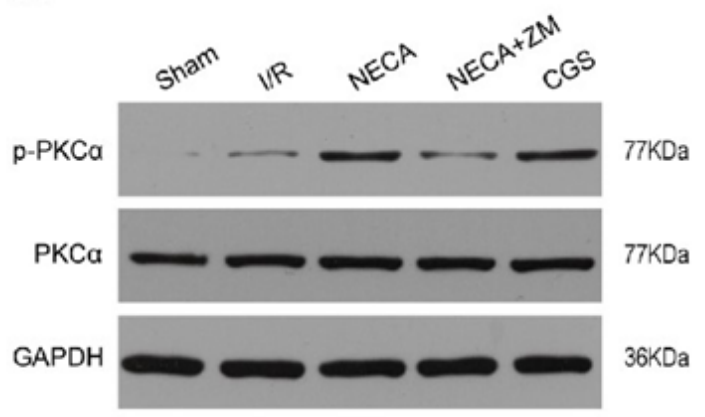

D
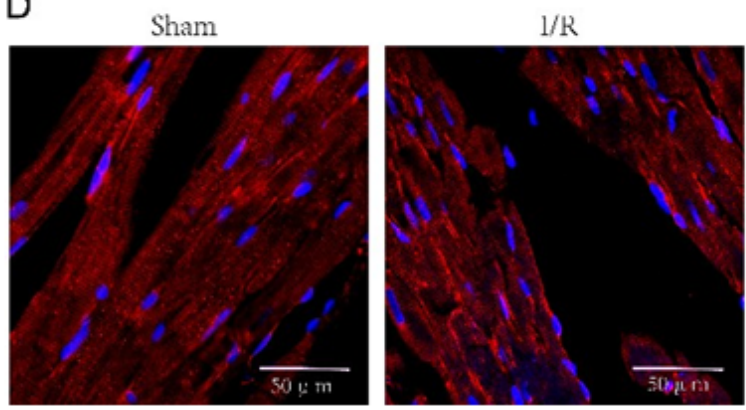

B

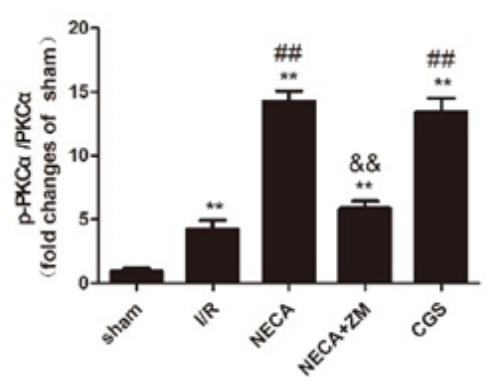

C

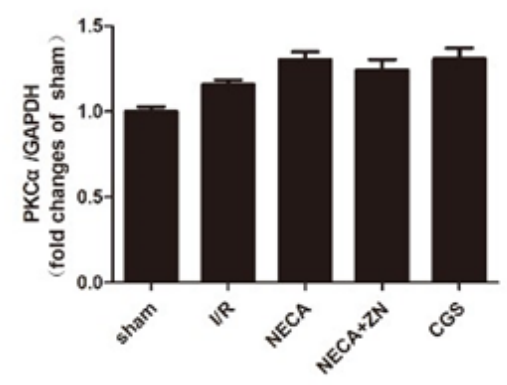

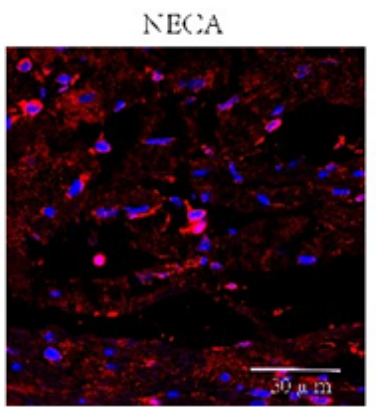
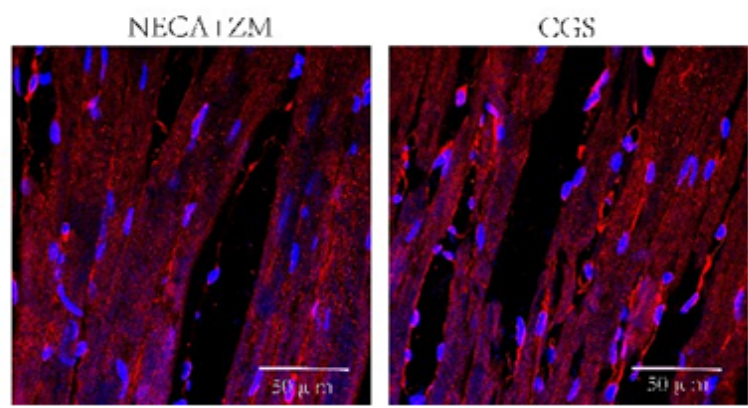

\section{Figure 6}

The effects of the NECA, NECA+ZM and CGS treatments on PKCa expression levels, phosphorylation and translocation. (A) Representative immunoblots of p-PKCa, PKCa and GAPDH in the different drug treatment groups. (B) p-PKCa expression. (C) Total PKCa expression. Data are expressed as mean $\pm S D$, $n=6 . * * P<0.01$ versus the sham group, $\# \# P<0.01$ versus the $1 / R$ group, $\& \& P<0.01$ versus the NECA group. (D) The localization of PKC-a. Representative photomicrographs of in situ detection of PKCa by indirect immunofluorescent staining. Blue fluorescence indicates nuclei; red fluorescence indicates PKCa; original $200 \times, n=5$. 


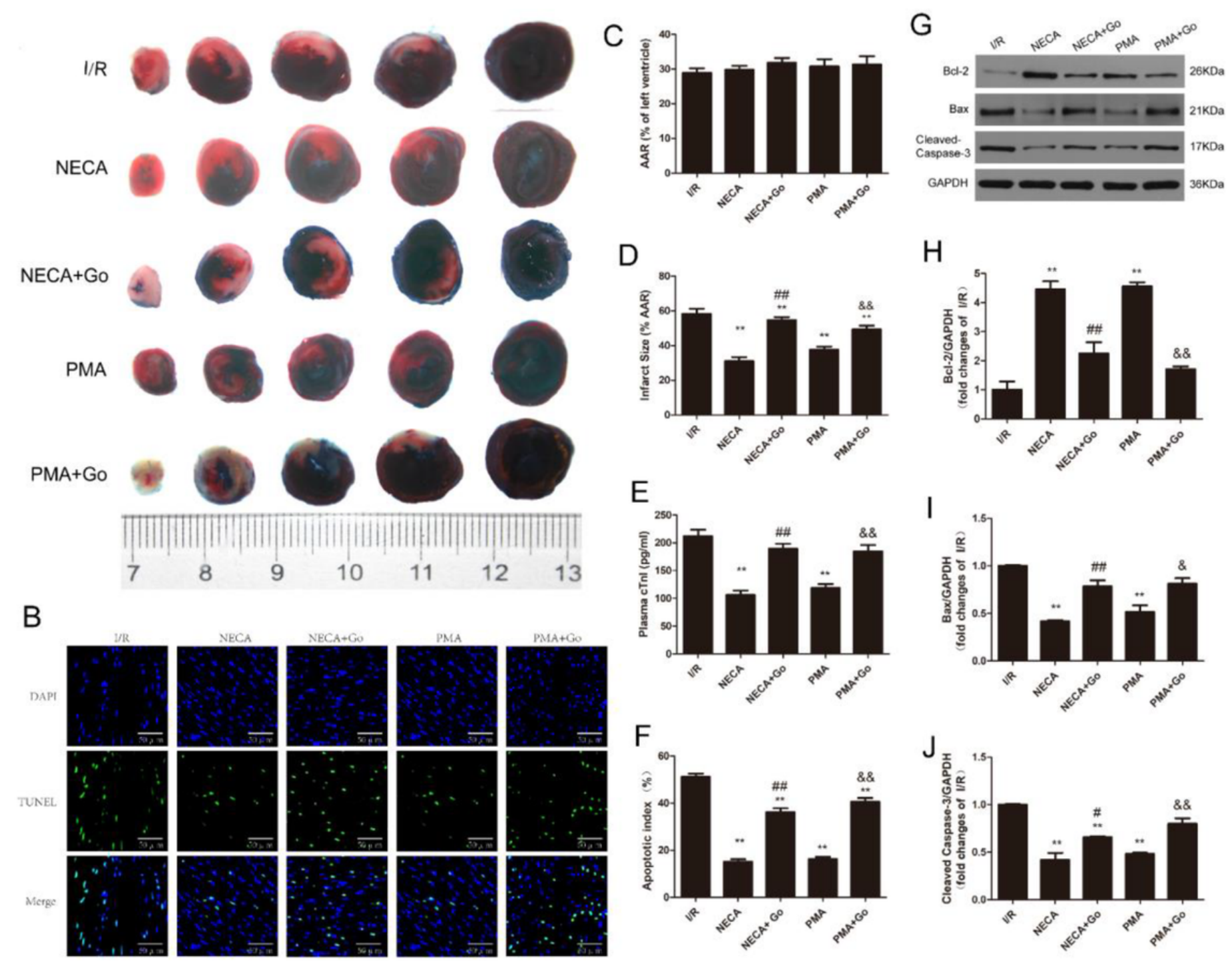

Figure 7

The effects of the NECA, NECA+Go, PMA and PMA+Go treatments on AAR, infarct size, Plasma cTnl level, apoptotic index and apoptotic signaling. (A) Representative image of cardiac slices determined by TTC and Evans blue staining, the blue-stained area represents the non-ischemic region; red-stained area represents ischemic but not infarcted portion; and the negative-stained area represents the infarcted portion. (B) Representative photomicrographs of in situ detection of apoptotic cardiomyocytes by TUNEL staining. Blue fluorescence indicates nuclei of total cardiomyocytes; green fluorescence indicates TUNELpositive nuclei; original 200×. (C) AAR expressed as a percentage of the total left ventricle ( $n=9)$. (D) Infarct size expressed as a percentage of the AAR(n=9). (E) Plasma cTnl concentrationn $(n=9)$. $(F)$ Percentage of TUNEL-positive nuclei(n=9). (G) Representative immunoblots of Bcl-2, Bax, Cleaved Caspase-3 and GAPDH in the different drug treatment groups. $(\mathrm{H}) \mathrm{Bcl}-2$ expression(n=6). (I) Bax expression( $n=6)$. (J) Cleaved Caspase-3 expression $(n=6)$. Data are expressed as mean $\pm S D, * * P<0.01$ versus the I/R group, \#P<0.05 versus the NECA group, \#\#P<0.01 versus the NECA group, \&P<0.05 versus the PMA group. \&\&P< 0.01 versus the PMA group. 
A

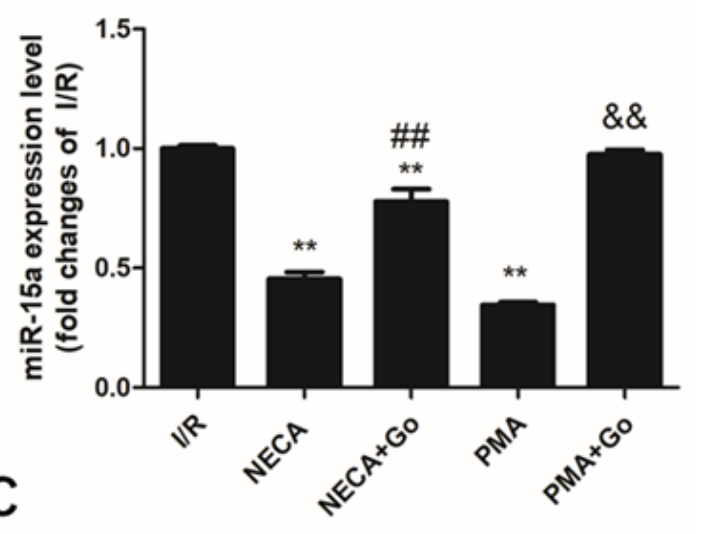

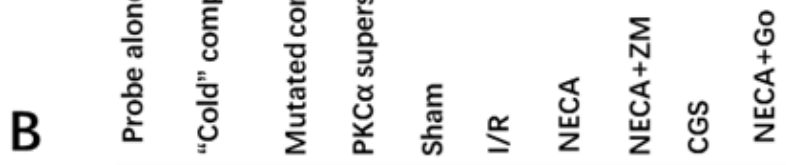

$\longleftarrow$ PKC $\alpha$ supershift

\section{5'‥CAGCACATAATGGTTTGTGGATTTTGAAAAGGTGCAGGCCATATTGTGCTGCG ‥3'}

PKC $\alpha$ binding site

\section{Figure 8}

The effects of NECA, NECA+Go, PMA and PMA+Go treatments on miR-15a expression. (A) the miR-15a expression in the different drug treatment groups. Data are expressed as mean $\pm S D, n=6$. ${ }^{*} P<0.01$ versus the I/R group, \#\#P< 0.01 versus the CGS group, \&\&P< 0.01 versus the PMA group. (B) Binding of PKCa to pri-miRNA-15a. For EMSA, a biotin-labeled pri-miRNA-15a oligonucleotide, containing the hypothesized PKCa binding site was used. As a competitor for this site, either 200×mutated or unlabeled ("cold") oligonucleotide was used to saturate the complex, A PKCa antibody was used for supershift analysis. (C) Predicated PKCa binding sequence including the subsequence we used for EMSA according to a previous study 

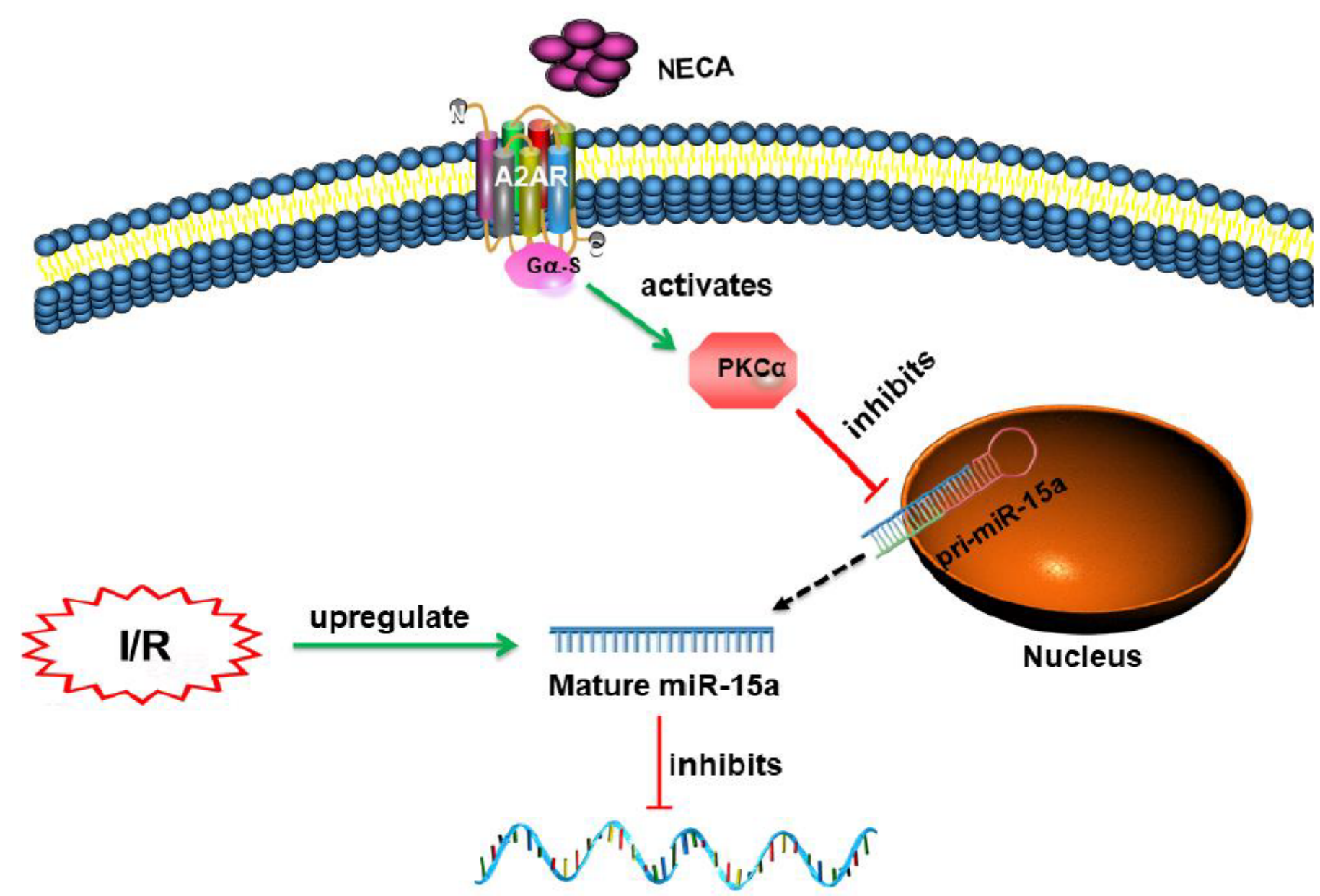

\section{Bcl-2 mRNA}

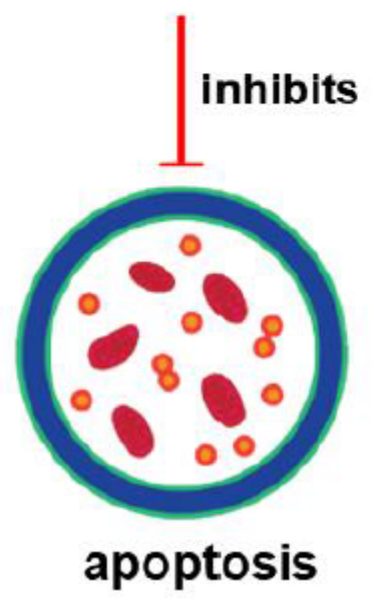

Figure 9

Proposed mechanisms of NECA attenuates myocardial ischemia/reperfusion injury in type 2 diabetic rats

\section{Supplementary Files}

This is a list of supplementary files associated with this preprint. Click to download. 
- Supplements.docx.pdf

Page 26/26 